\title{
Curvature-modulated phase separation in lipid bilayer membranes
}

Raghuveer Parthasarathy*, Cheng-han Yu*, and Jay T. Groves

* These authors contributed equally to this work

\section{Supporting Information: Three Figures}
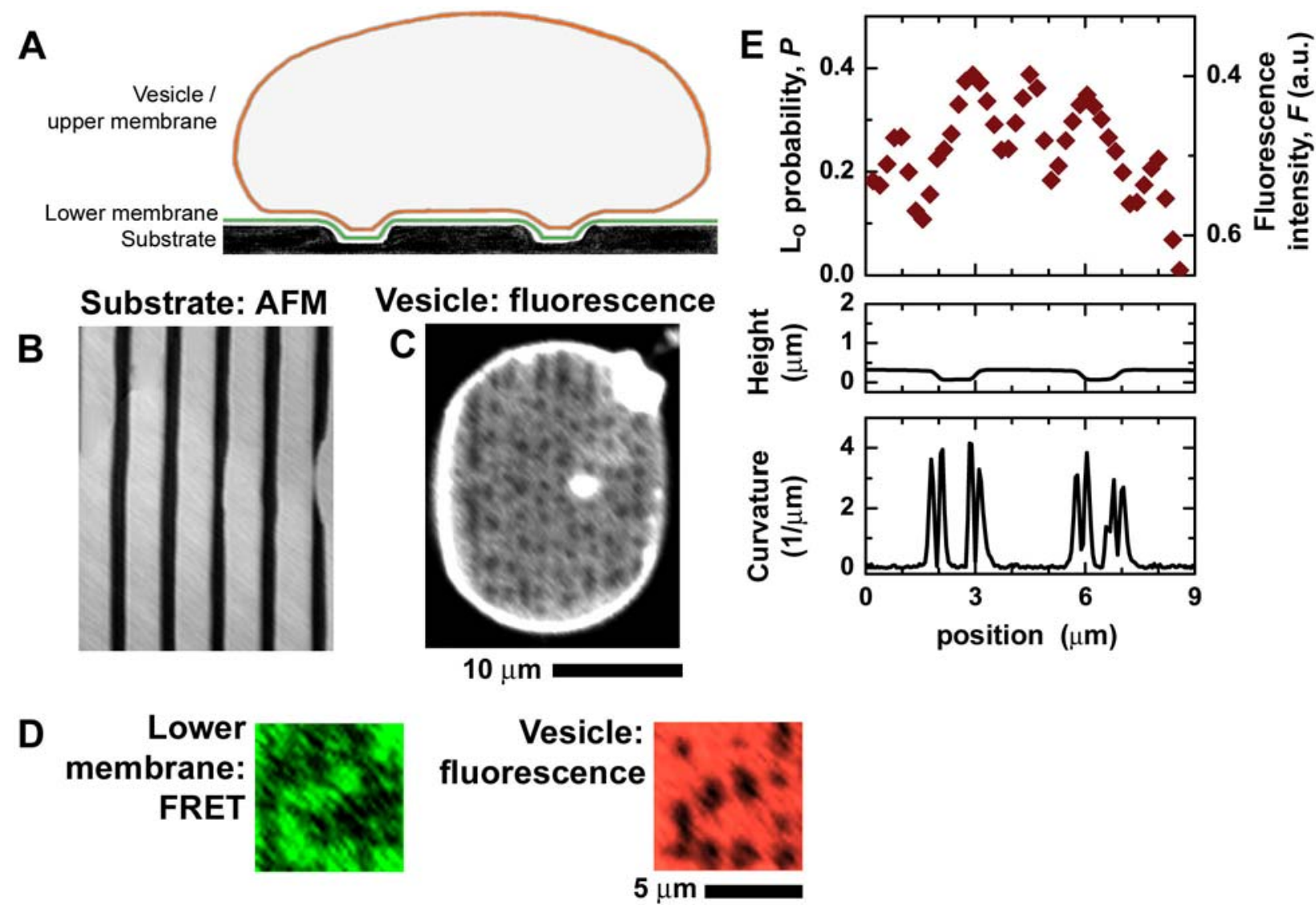

Figure S1. Curvature controls spatial organization of phase separation in intact lipid vesicles. (A) Schematic illustration of the experimental setup: a topographically patterned quartz substrate, a supported lipid bilayer membrane (green), and a giant lipid vesicle (red), whose lower surface is in contact with the supported membrane. (B) Atomic force micrograph of the substrate underlying the bilayers of (C-D), consisting of wide parallel grooves. (C) Fluorescence image of a giant unilamellar vesicle, focused on its lower membrane. Phase separation into liquid-ordered $\left(\mathrm{L}_{0}\right.$, dark) and liquid-disordered $\left(\mathrm{L}_{\mathrm{d}}\right.$, bright) phases has occurred, with $\mathrm{L}_{0}$ domains aligned along the topographic pattern. (D) Fluorescence image of corresponding sections of the supported lipid bilayer (green) and vesicle membrane (red). Non-uniform green intensity due to FRET, the inverse of the red fluorescence image, indicates continuous intermembrane contact (as discussed in the main text). (E, upper graph) Relative $\mathrm{L}_{0}$ domain probability ( $P$, see main text) and fluorescence intensity $(F)$ from a subset of $(C)$, averaged over the direction parallel to the substrate stripes. (E, middle graph) The substrate height profile, measured by atomic force microscopy. (E, lower graph) The local curvature of the substrate. The five preferred positions of $L_{o}$ domains (maxima in the upper graph) correspond to the five low-curvature regions located at the centers of the plateaus and the centers of the wide grooves. 


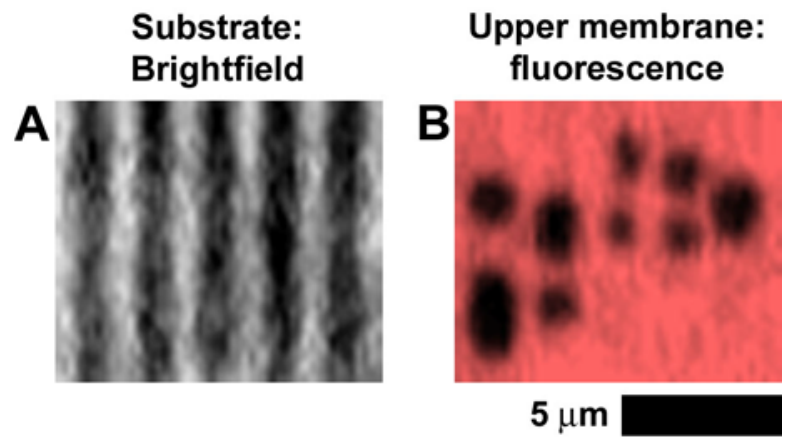

Figure S2. Alignment of upper membrane $\mathrm{L}_{0}$ domains with substrate-imposed curvature patterns for DOPC/Egg sphingomyelin (ESM)/cholesterol membranes. (A) Brightfield image of the substrate, similar to the substrate of Fig.1. (B) Fluorescence image of the phase-separated upper membrane. Though curvature-controlled domain alignment occurs, upper membranes of the composition 1:1:1 DOPC:ESM:cholesterol (a popular membrane raft model) typically do not homogenize upon giant vesicle rupture (see Fig. 1). These membranes have high miscibility transition temperature relative to the ambient temperature (a difference of over $30^{\circ} \mathrm{C}$ ), compared to the membranes discussed in the main text $\left(<10^{\circ} \mathrm{C}\right)$, leading to a large line tension that holds domains together. 


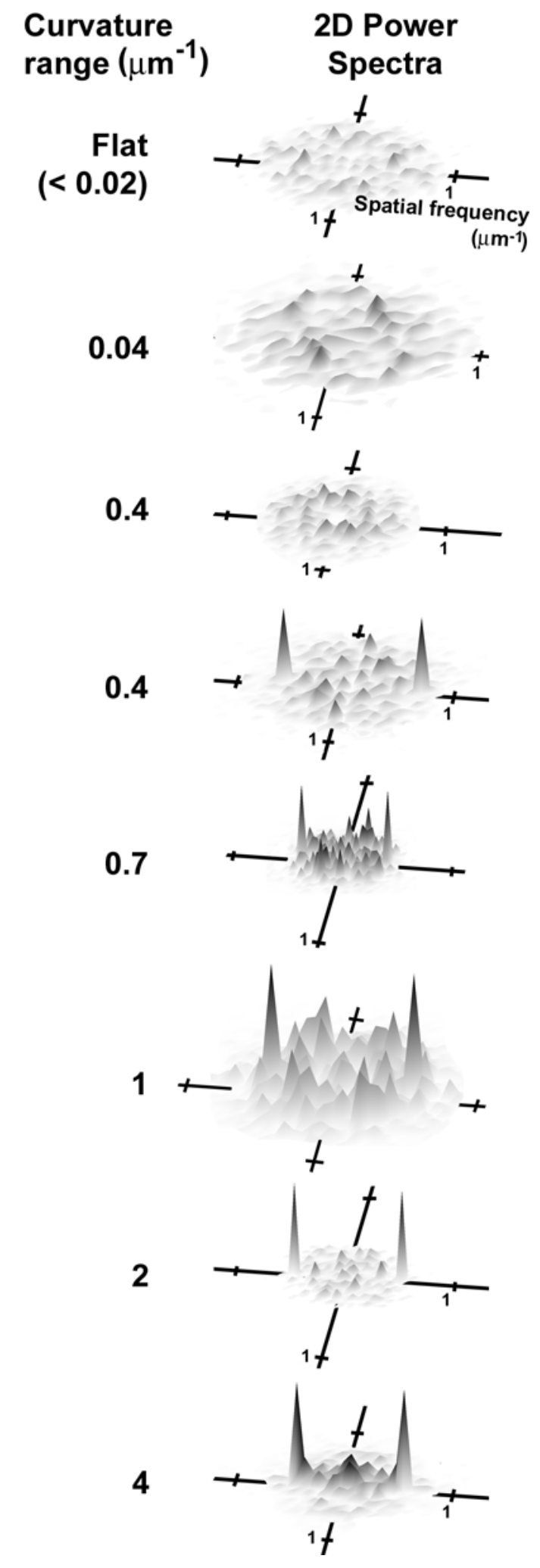

Figure S3. Power spectra of upper membrane fluorescence images from membranes on substrates with different curvature ranges. Three data sets are reproduced from Figure 3. The topmost spectrum is from an unpatterned (flat) substrate that has curvature variations of at most $0.02 \mu \mathrm{m}^{-1}$. Beyond a critical minimum curvature $\left(c_{\min }=0.8 \pm 0.2 \mu \mathrm{m}^{-1}\right)$, the upper membrane domains are always spatially periodic in response to the periodic substrate-imposed curvature, as evidenced by peaks in the spectra corresponding to the periodicity of the substrate. 